\title{
Quantifying the quantum
}

\section{Stephan Schlamminger looks at the origins of the Planck constant and its current role in redefining the kilogram.}

A child on a swing is an everyday approximation of a macroscopic harmonic oscillator. The total energy of such a system depends on its amplitude, while the frequency of oscillation is, at least for small amplitudes, independent of the amplitude. So, it seems that the energy of the system can be adjusted continuously from zero upwards - that is, the child can swing at any amplitude. This is not the case, however, for a microscopic oscillator, the physics of which is described by the laws of quantum mechanics. A quantum-mechanical swing can change its energy only in steps of $h v$, the product of the Planck constant and the oscillation frequency - the (energy) quantum of the oscillator.

In 1901, the idea of energy quantization was put forward by Max Planck ${ }^{1}$. The year before, Planck had published an article wherein he predicted the spectral shape of black-body radiation (the radiation emitted from a perfect absorber). However, better experimental evidence became available, with which Planck's initial theory was discrepant. So he attempted another calculation and used an 'audacious assumption': energy quantization. A black body can be described as a distribution of oscillators, the energy of which Planck required to be the product of an integer, a new constant $h$, and the oscillator's frequency. Using elementary combinatorics and Boltzmann's entropy equation, he managed to obtain an equation for the spectral shape of the black-body radiation that matched the experimental observations perfectly. From the published spectral data and his theory, Planck estimated a value of $6.55 \times 10^{-34} \mathrm{~J}$ s for $h$. Today, the accepted value for the Planck constant is $6.626070040 \times 10^{-34} \mathrm{~J} \mathrm{~s}$, with a relative uncertainty of $1.2 \times 10^{-8}$.

The unit of the Planck constant, J s, can be written as $\mathrm{kg} \mathrm{m}^{2} \mathrm{~s}^{-1}$. This equivalence hints at an interesting idiosyncrasy in the present version of the International System of Units (SI): to measure a quantum mechanical constant $(h)$ with high precision, a macroscopic experiment is necessary, because the unit of mass, the kilogram, can only be accessed with high precision at the one-kilogram value through its definition as the mass of the International Prototype of the Kilogram (IPK). The IPK is the only object on Earth whose mass we know for certain $^{2}-$ all relative uncertainties increase from thereon.

The revised SI, likely to come into effect in 2019, is based on fixed numerical values, without uncertainties, of seven defining constants, three of which are already fixed in the present SI; the four additional ones are the elementary charge and the Planck, Boltzmann and Avogadro constants. Any experiment that can be used in the present SI to measure the Planck constant from known calibration masses can be operated backwards in the revised SI: it would measure mass based on a fixed, defined value of $h$. Such an experiment translates between the quantum and the macroscopic world ${ }^{3}$.

One experiment that connects $h$ to the kilogram involves a balance invented in 1975 by Bryan Kibble 4 . At the heart of the Kibble balance, formerly known as the watt balance, is a coil in a magnetic field that can operate as both a motor and a generator. For the former, a current $I$ is passed through the coil, resulting in a force $F$. For the latter, the coil is swept with velocity $v$ through the field, producing an induced voltage $U$ at the coil's terminals. Kibble realized that due to the symmetry in Maxwell's equations, the quotient of force and current, $F / I$, is identical to the quotient of voltage and coil velocity, $U / v$, if the velocity and force point in the same direction. This principle enables one to equate both quotients and hence measure a

force - in this case the weight of a mass $m$ - as the product of a current and a voltage divided by a velocity, $m g=U I / v$ (with $g$ the Earth's gravitational acceleration).

Meanwhile, the discoveries of the Josephson and quantum Hall effects

led to precise electrical measurements involving the Planck constant and the elementary charge ${ }^{5}$. For the product of voltage and current UI, the elementary charge cancels out; it only depends on the Planck constant, two microwave frequencies, $f_{1}$ and $f_{2}$, used for the Josephson voltage standard, and a known numerical factor $r$, which depends on the experimental setup. Hence, the mass can be expressed as $m=h r f_{1} f_{2} / g v$.

Replacing a century-old artefact with a set of fundamental constants and recipes to obtain the unit of mass from these constants and the Kibble balance brings the SI closer to a system of units that is "for all time, for all people".

\section{STEPHAN SCHLAMMINGER is at the} Physical Measurement Laboratory at the National Institute of Standards and Technology, 100 Bureau Drive, Gaithersburg, Maryland 20899-8171, USA.

e-mail: stephan.schlamminger@nist.gov

\section{References}

1. Planck, M. Ann. D. Phys. 4, 553-563 (1901).

2. SI Brochure: The International System of Units (SI) 8 th edn (BIPM, 2014); http://www.bipm.org/en/publications/si-brochure/

3. Haddad, D. et al. Metrologia 53, A83-A85 (2016).

4. Kibble, B. P. in Atomic Masses and Fundamental Constants Vol. 5 (eds Sanders, J. H. \& Wapstra, A. H.) 545-551 (Springer, 1976)

5. Piquemal, F. Nat. Phys. 12, 284 (2016).

$\mathrm{m} e \AA \mathrm{s} \mathrm{u} R E_{\mathrm{h}}$

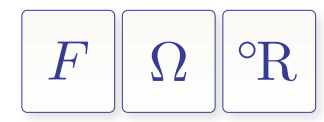

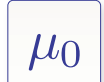

$\varepsilon_{0}$

$\alpha$

$\sigma$

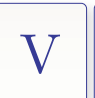

$\mathrm{R}$ 